F or almost 50 years, exploration of the large Galilean moons of Jupiter has delivered a continuing stream of surprises. The four moons - Io, Europa, Ganymede and Callisto - have increasingly come to appear as an exotic and remarkably diverse planetary system in their own right. In 1979 we had the discovery of the most dramatic volcanism known in the Solar System, on the innermost Galilean satellite
Io, during the Voyager space mission ${ }^{1}$; and in 1996 the Galileo spacecraft revealed that Ganymede has a strong magnetic field ${ }^{2}$, probably stemming from an internal dynamo.

Now, in the paper on page 777 of this issue $^{3}$, comes strong evidence for the existence of oceans not very far below the surfaces of Europa and Callisto. This evidence results from the observation of electromag-

\title{
The geometry of adaptation
}

Nick Barton

$\mathrm{D}$ arwin $^{1}$ explained the evolution of complex adaptations, such as the vertebrate eye, through the gradual "accumulation of innumerable slight variations, each good for the individual possessor". In contrast, the early geneticists emphasized the role of major mutations, and disregarded natural selection ${ }^{2}$. The mathematical theory of Fisher, Haldane and Wright reconciled Mendelian genetics with Darwinian gradualism by showing that genetic variants that slightly enhanced reproductive success would quickly replace their competitors. Nevertheless, the conflict over the magnitude of the genetic changes responsible for adaptation continues to this day ${ }^{3}$. Although classical population genetics can find the consequences of a given pattern of selection, it says nothing about the causes of such selection. Indeed, theoretical support for gradualism rests mainly on a geometrical analogy, proposed by Fisher ${ }^{4}$ in 1930, which suggests that large changes to a complex system are unlikely to be favourable. Now, reporting in Evolution, H. Allen Orr ${ }^{5}$ shows that adaptation may be based on larger factors than previously supposed.

Fisher's argument is simple. Suppose that an organism is described by $n$ traits, and that its fitness increases towards some optimal value. If a population lies away from the optimum, then a random change that takes it inside an $n$-dimensional sphere centred on the optimum will increase its fitness, and may be established by natural selection (Fig. 1, overleaf). A small change is as likely to be favourable as unfavourable, whereas a change larger than the diameter, $d$, of the sphere must be disadvantageous. Fisher showed that, for large $n$, the chance that a change of magnitude $r$ (a sum over all traits) increases fitness is $(1-\Phi(x))$, where $\Phi$ is the cumulative normal distribution, and $x=r \sqrt{n} / d$ is a measure of the effective size of the change. So, as the complexity of the organism (represented by $n$ ) increases, a change of absolute magnitude $r$ is less likely to be an improvement.

But a fundamental flaw in Fisher's argument was found, half a century later. Kimura ${ }^{6}$ pointed out that although small changes are most likely to increase fitness, they are also most likely to belost by chance. Even in a large population, a single mutation that increases relative fitness by $s$ has a chance of only around $2 s$ of being established ${ }^{4}$. Kimura showed that if the selective advantage of a mutation is proportional to its effective size, $x$, then the chance that the mutant is favourable and becomes common is $2 x(1-\Phi(x))$. The expected effective size of successful mutations is $E[x]=4(\sqrt{2 / \pi}) / 3=$ 1.06. Thus, mutations of intermediate effect are most likely to contribute. For example, with $n=50$ dimensions, the first adaptive step will average $30 \%$ of the distance to the optimum, $d / 2$. However, because its direction is random, it will take the population only about $4 \%$ further towards the optimum.

Orr ${ }^{5}$ now goes beyond these arguments by considering the whole evolutionary sequence by which a population approaches the optimum. The distribution of sizes of the first step is given by Kimura's formula. The population is then, on average, a factor $(1-2 E[x] / n)$ closer to the optimum. The process is then repeated from this new starting point. Orr shows that, to a close approximation, substitutions continue with a distribution that retains the same form, but has a continually shrinking scale (Fig. 1). Remarkably, the overall distribution of sizes of factors fixed over this adaptive 'walk' to the optimum is exponential, $\sim \exp (-\lambda x)$, with $\lambda=2.9$. This result is independent of the dimension $n$, but does assume a uniform distribution of mutational effects.

Orr shows that for a variety of mutational distributions, biased either to smaller or larger effects, the distribution of factors fixed remains close to exponential (although with larger or smaller $\lambda$ ). Evolution by a series of gradually diminishing random draws means that the largest step, $x_{\max }$, is probably larger than the first, and increases logarithmically with $n$; for example, with $n=50$ dimensions, $E\left[x_{\max }\right]=1.68$, corresponding to a magnitude $48 \%$ of the original distance to the optimum. Thus, large changes are established in the early stages of adaptation towards a fixed optimum, mainly because alleles with the

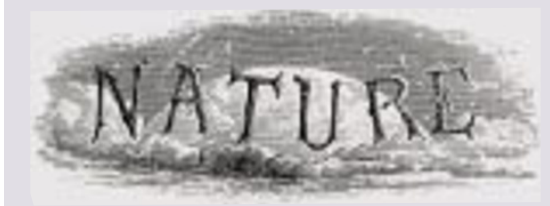

100 YEARS AGO

What was the condition of England in 1845? Her universities had degenerated into hauts lycées. With regard to the University teaching, I may state that even as late as the late fifties a senior wrangler - I had the story from himself - came to London from Cambridge expressly to walk about the streets to study crystals, prisms and the like in the optician's windows. Of laboratories in the universities there were none; of science teaching in the schools there was none; there was no organisation for training science teachers. If an artisan wished to improve his knowledge he had only the moribund Mechanics' Institutes to fall back upon. ... We lacked then everything which Germany had equipped herself with in the matter of scientific industries. Did this matter? Was it more than a mere abstract question of a want of perfection? It mattered very much! ... At this time we had, fortunately for us, in England, in very high place, a German fully educated by all that could be learned at one of the best equipped modern German Universities, where he studied both science and the fine arts. I refer to the Prince Consort. From that year to his death he was the foundation of our English educational renaissance ... From Nature 20 October 1898.

\section{YEARS AGO}

Co-operation between the Standard Oil Co. and its subsidiaries of America and the I.G. Farben-industrie of Germany led to the formation in 1929 of a joint company ... . The final result of this cooperation brought to America the technique of the preparation of Buna $S$, an all-purpose synthetic rubber ... .

Howard tells with what sense of urgency he and his colleagues regarded the necessity for the setting up of a synthetic rubber industry, and how, in the face of a general apathy, his Company decided to erect the necessary plant for the manufacture of Buna $S$ on a small scale. Even after Pearl Harbour, all their efforts to get this vital material made on a large scale came to nothing ... until the setting up of the Baruch Committee in August 1942; and the resulting appointment of a rubber director with absolute power cleared away all the difficulties, with the result that in a very short time the American synthetic rubber industry was among the largest in the country. From Nature 23 October 1948. 


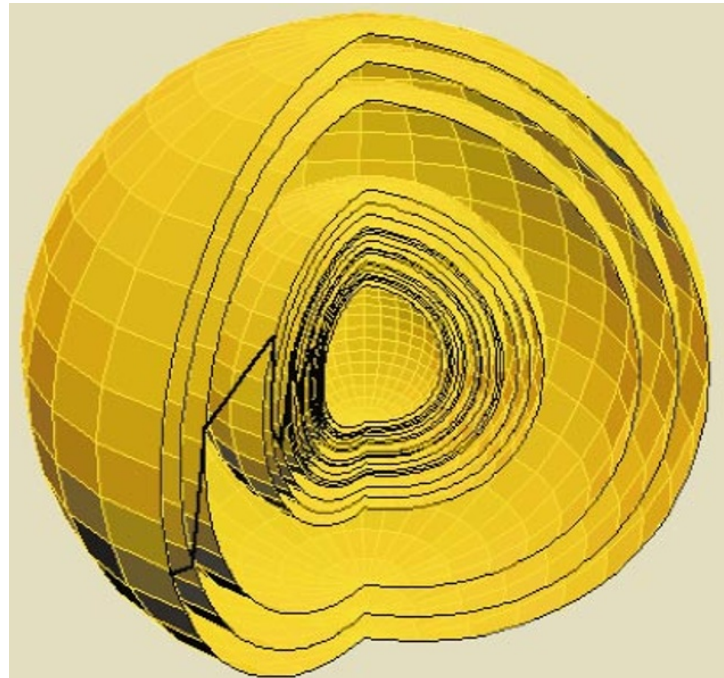

Figure 1 Approach of a population towards an optimum through selection of a series of random mutations, through a space of ten dimensions. The population begins at a point on the outer sphere, at a distance $d / 2$ from the central optimum (where $d$ is the diameter of the sphere). The first successful mutation has magnitude $r=0.137 d$, and takes the population $8.7 \%$ of the way to the optimum (first line, leading to the second sphere). The third successful mutation has the largest magnitude, $0.271 d$; it is followed by smaller steps which, on average, follow a geometric series.

largest effect on fitness are most likely to be picked up by natural selection. Later, small changes do contribute, but they are not responsible for much of the improvement in fitness.

The prediction that the effects of the genes responsible for adaptation should follow an exponential distribution roughly applies to individual traits, as well as to the sum over all traits. Orr argues that studies ${ }^{7}$ of the quantitative trait loci (QTL), which distinguish close taxa, are consistent with this prediction. But it is not clear what would be predicted by other models of evolution, or whether these could be distinguished. Existing methods for detecting QTL are biased towards finding genes of spuriously large effect $^{7}$. More convincing evidence for the importance of major genes comes from studies of 'candidate loci', which often contribute to divergence and standing variation ${ }^{8,9}$. However, such examples hardly fit with Fisher's model, whereby mutations are drawn randomly from any of the many genes that might influence a trait.

Some functions may necessarily be composed of many minor changes. For example, the efficiency and accuracy of translation can be improved by using triplet codons that match the most abundant transfer RNAs. Each such change could have only a tiny effect on fitness ${ }^{10}$, so, for codon-usage bias, adaptation is based on minute variations. Nevertheless, such examples are hard to find and there are many limits to the success of weakly selected variants, beyond the low fixation probability identified by Kimura ${ }^{6}$ and Orr $^{5}$. In a large, asexual population, a favourable allele can fix only if it confers an advantage that outweighs the loss in fitness owing to the deleterious alleles with which it arises ${ }^{4}$. Even with sexual reproduction, linkage reduces the effectiveness of natural selection, and can greatly reduce the fixation probability of a weakly favoured allele ${ }^{11}$. Indeed, bias in codon usage is weaker in regions of reduced recombination in Drosophila ${ }^{12}$.
Fisher's geometrical analogy is an extreme abstraction of organismic complexity. One problem is that it is hard to know what is meant by the number of dimensions, $n$. The morphology, development and behaviour of an organism can be described by an indefinite number of phenotypic dimensions that may all be influenced by many genes. The number of degrees of freedom, $n$, is presumably limited by the number of genetic loci $\left(\sim 10^{5}\right.$ genes and $\sim 3 \times 10^{9}$ base pairs in man). However, once one thinks in terms of gene sequences rather than continuous traits, a different geometry is required. Although many sequences are possible $\left(4^{k}\right.$ with $k$ bases), only a few $(\sim 3 k)$ can be reached by a single mutation. It may be possible to do an analysis similar to Orr's for this tightly restricted geometry ${ }^{13}$, although this might come to different conclusions. The issues raised by Orr's work are relevant to the optimization of any complex structure - be it computer software or a mechanical device. But the universal genetic code, together with simple mathematics of natural selection, may allow population genetics to provide a more general understanding of adaptation than is possible in other contexts.

Nick Barton is in the Institute of Cell, Animal and Population Biology, King's Buildings, University of Edinburgh, Edinburgh EH9 3JT, UK.

e-mail:n.barton@ed.ac.uk

\footnotetext{
1. Darwin, C. On the Origin of Species by Means of Natural Selection (Murray, London, 1859).

2. Provine, W. The Origins of Theoretical Population Genetics (Univ. Chicago Press, 1971).

Orr, H. A. \& Coyne, J. A. Am. Nat. 140, 725-742 (1992).

4. Fisher, R. A. The Genetical Theory of Natural Selection (Oxford Univ. Press, 1930).

5. Orr, H. A. Evolution 52, 935-949 (1998).

6. Kimura, M. The Neutral Theory of Molecular Evolution (Cambridge Univ. Press, 1983).

7ynch, M. \& Walsh, J. B. Genetics and Analysis of Quantitative Traits (Sinauer, Sunderland, MA, 1998).

8. McKenzie, J. A. \& Batterham, P. Trends Ecol. Evol. 9, 166-170 (1994).

9. Long, A. D., Mullaney, S. L., Mackay, T. F. C. \& Langley, C. H. Genetics 144, 1497-1510 (1997).

10. Bulmer, M. Genetics 129, 897-907 (1991).

11. Barton, N. H. Genetics 140, 821-841 (1995).

12. Kliman, R. M. \& Hey, J. Genetics 137, 1049-1056 (1994).

13. Gillespie, J. H. Evolution 38, 1116-1129 (1984).
}

\section{Daedalus \\ Dead metallic silence}

Noise, that curse of modern living, is spread in many ways. The racket of a diesel engine, for example, is greatly amplified by vibrating sheet-metal surfaces such as the rocker cover, the sump-case and the body panels of the vehicle housing it. Workplace machines, domestic gadgets, and even clattering pots and pans, also owe much of their characteristic noise to the undamped vibration of metal surfaces. If all our products could be made (say) of wood, life would be much more peaceful.

So Daedalus wants to load engineering metals with vibration-damping inclusions. Cast iron, for example, one of the quietest of metals, contains many tiny graphite inclusions which absorb vibration. Sadly, they also act as crack-initiating sites, and make it brittle. Daedalus recalls that polystyrene (which has rather a metallic ring itself) is damped by certain organometallic ring compounds. The organic ring in these molecules can rotate stepwise around the bond joining it to the metal. The polymer is strongly damped in the acoustic range of frequencies corresponding to the stepping rate of the ring.

So DREADCO chemists are synthesizing organic ring molecules capable of binding to iron and other engineering metals, and are using powder sintering to disperse the most likely candidates into bulk metals. Cunningly, they are seeking molecules that are the stablest combination of that set of atoms. Trapped in its metallic cage at the sintering temperature, such a molecule might well decompose; but it would re-form again when the metal cooled. The resulting metals will be acoustically dead yet still tough and ductile - single molecules are far too small to act as crack initiators.

When perfected, DREADCO's 'Dead Metal' range of alloys should sweep through society. The big noises, such as diesel engines, are the obvious targets. But Daedalus attributes much of the edginess of modern living to the quieter but everpresent background noise from centralheating systems, air-conditioning units, office equipment, refrigerators, cookers, washing machines and so on. The formless, ever-changing insistence of this endless accompaniment to life gets on our nerves in some very subtle way. He recalls the story of a housewife in a modern kitchen who, hearing a sudden change in its complex background racket, asked in bewilderment, "What's just stopped?". With Dead Metal, they will all stop. David Jones 